\title{
Severe Jabbing Headache Associated with Airplane Travel
}

\author{
Han-Joon Kim, Yong-Jin Cho, Joong-Yang Cho, Keun-Sik Hong
}

Can. J. Neurol. Sci. 2008; 35: 267-268

Although the second edition of The International Classification of Headache Disorders includes diagnostic criteria for several new clinical entities, ${ }^{1}$ it does not encompass all types of headaches encountered in clinical practice.

In 2004, a new type of headache called 'airplane headache,' which is characterized by severe jabbing pain in the head during altitudinal changes in airplane travel, was described. ${ }^{2}$ Since its initial description, seven more patients with 'airplane headache' have been described in two reports. ${ }^{3,4}$ All of the patients exhibited very stereotypical symptoms. However, although pathology in the paranasal sinuses has been suggested, ${ }^{3}$ radiologic abnormalities to support this pathology have not been reported.

In this report, we describe a patient with abnormal CT findings who suffered from 'airplane headache' for 15 years.

\section{CASE RePORT}

A 38-year-old man presented with headache associated with airplane travel. His past medical history was unremarkable, and he was not taking any medication. He experienced his first headache attack 15 years ago while on an airplane. When the plane began to descend, he suddenly developed a severe, sharp and jabbing headache in the right supraorbital area. The headache was not accompanied by nausea, vomiting, photophobia, phonophobia, autonomic symptoms, eyeball pain, or visual disturbances. His headache was not alleviated or aggravated by changing the position of the head. The headache lasted for about 30 minutes and resolved spontaneously immediately after landing.

Since then, he has experienced recurrent headaches with the same characteristics during about 80 percent of his flights. The headache attacks only occurred during descent and usually lasted for about 30 minutes. On rare occasions, the headache would spontaneously disappear immediately after onset. Drinking coffee during the flight did not prevent or alleviate the patient's headache. He did not attempt to use medication to relieve the headache symptoms on any occasion during the 15 years he had been experiencing the headaches. After his most recent flight, although the severe jabbing headache resolved after landing, he continued to experience a headache of moderate severity in the right frontal area that lasted for four days and then disappeared. He visited the hospital due to this change in headache pattern.

His body weight was $64 \mathrm{~kg}$, and his height was $170 \mathrm{~cm}$. The findings of general physical and neurological examinations were normal. He had no signs or symptoms to suggest paranasal sinus disease. A simple X-ray of the paranasal sinuses showed normal findings. Cranial CT revealed no abnormalities except for mucosal thickening of the right ethmoid sinus (Figure).

\section{Discussion}

Eight cases of recurrent headaches associated with airplane travel have been reported to date..$^{2-4}$ All of the patients were men, and the age of onset ranged from 20 to 40 years. The headaches were localized in the periorbital area: on the right in six, on the left in one, and along the midline and forehead in one patient. The pain was severe, sharp, and jabbing, and it usually lasted for about 15 to 20 minutes. Five patients experienced headaches during descent, two experienced headaches during or after ascent and one had headaches during both ascent and descent. Only two patients had accompanying autonomic symptoms. The disease duration ranged from seven months to eight years. Radiologic evaluation showed no relevant abnormality in any patient.

Our patient had many characteristics in common with the previously reported cases, including male gender, right supraorbital location, and headache onset during descent. One notable feature of our case was the long duration of the disease, which suggests that this type of headache can persist for a long period of time without exacerbation or remission.

The pathophysiology of this type of headache is uncertain. The fact that the headache occurred only during rapid changes in altitude suggests that changes in ambient pressure play a key role in the development of these headaches. Barotrauma has been suggested as the most probable cause of this type of headache. ${ }^{3}$ In patients with pre-existing obstruction of the paranasal sinus due to sinusitis, airplane descent will lower the relative pressure within the sinus air cells. This may cause a vacuum effect and lead to tension in the mucosal lining of the sinus, resulting in mucosal damage and pain. Indeed, a unilateral severe headache

From the Department of Neurology, Inje University Ilsan Paik Hospital, Goyang-si, Gyeonggi-do, Republic of Korea.

Received July 31, 2007. Final Revisions Submitted November 28, 2007. Reprint requests to: Han-Joon Kim, Department of Neurology, Inje University Ilsan Paik Hospital, 2240, Daewha-dong, Ilsanseo-gu, Goyang-si, Gyeonggi-do, 411-706, Republic of Korea 


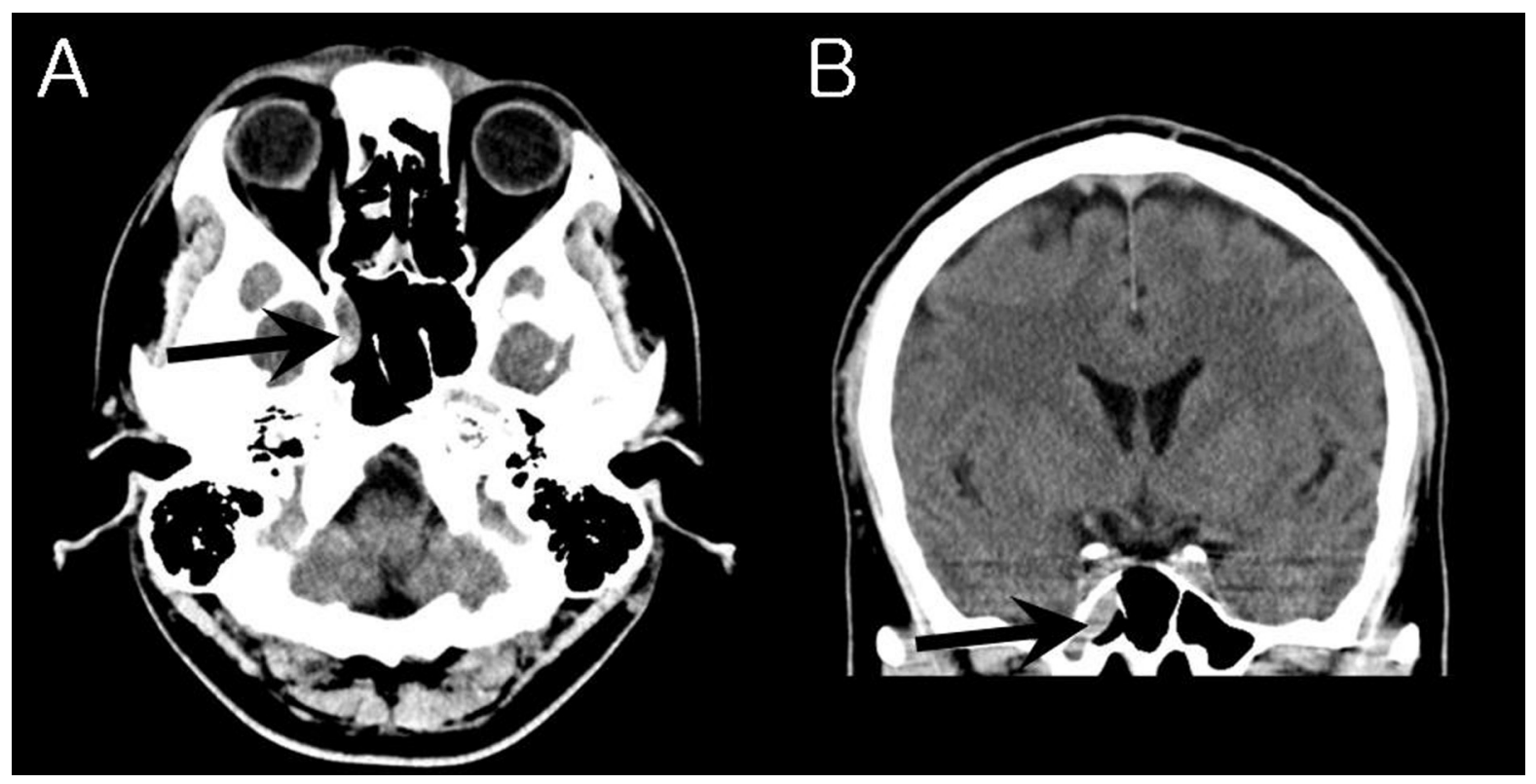

Figure. Axial (A) and coronal (B) cranial CT scans show mucosal thickening of the right ethmoid sinus (arrows).

during airplane descent was described in a patient with radiologically-proven frontal sinus barotrauma. ${ }^{5}$ Barotrauma to the mucosa of the ethmoid sinus was implicated in the pathology of headaches associated with airplane travel $^{3}$ because mucosal changes in the ethmoid sinus could cause severe pain in the supraorbital area, ${ }^{6}$ which is where most of these patients experience pain. However, otorhinolaryngologic evaluation and imaging of the sinuses showed normal findings and failed to locate a relevant lesion, which was attributed to the delay in presentation to a clinic after headache attack and possible resolution of the sinus pathology.

Interestingly, cranial CT of our patient showed thickening of the right ethmoid sinus mucosa, which was expected but not found in previous cases. A relevant lesion may have caused the headache. However, because such a slight thickening of the mucosa is very common in asymptomatic individuals, we cannot exclude the possibility that the sinus mucosal changes on CT were not related to the development of this headache and were only an incidental finding. Unilaterality, the jabbing nature of the headache, and the autonomic symptoms in some patients suggest that this type of headache might share a common pathophysiology with cluster headaches.

There has been no report on the treatment of this type of headache. We assume that this type of headache is underrecognized, considering that many patients in the previous reports, including our patient, delayed seeking medical advice for many years.

\section{REFERENCES}

1. Headache Classification Committee of the International Headache Society. The International Classification of Headache Disorders. Cephalalgia. 2004;24:1-160.

2. Atkinson V, Lee L. An unusual case of an airplane headache. Headache. 2004;44:438-9.

3. Berilgen MS, Muengen B. Headache associated with airplane travel: report of six cases. Cephalalgia. 2006;26:707-11.

4. Mainardi F, Lisotto C, Palestini C, Sarchielli P, Maggioni F, Zanchin G. Headache attributed to airplane travel ("airplane headache"): first Italian case. J Headache Pain. 2007;8:196-9.

5. Segev Y, Landsberg R, Fliss DM. MR imaging appearance of frontal sinus barotrauma. Am J Neuroradiol. 2003;24:346-7.

6. Clerico DM. Sinus headaches reconsidered: referred cephalgia of rhinologic origin masquerading as refractory primary headaches. Headache. 1995;35:185-92. 